\section{References}

1. Kuper H, Polack S, Limburg H. Rapid assessment of avoidable blindness. Community Eye Health. 2006;19:68-9.

2. Eagan SM, Jacobs RJ, Demers-Turco PL. Study of luminance effects on pinhole test results for visually impaired patients. Optom Vis Sci. 1999;76:50-8.
3. Sun JK, et al. Visual acuity testing using autorefraction or pinhole occluder compared with a manual protocol refraction in individuals with diabetes. Ophthalmology. 2011;118:537-42. https://doi.org/ 10.1016/j.ophtha.2010.07.022

4. Loewenstein JI, Palmberg PF, Connett JE, Wentworth DN. Effectiveness of a pinhole method for visual acuity screening. Arch Ophthalmol. 1985;103:222-3.

\title{
Time to drop the phenylephrine from the paediatric cycloplegia protocol: informing practice through audit
}

\author{
Sue Llewellyn ${ }^{1,2} \cdot$ Payal Khandelwal $^{2,3} \cdot$ Selina Glaze ${ }^{2,3} \cdot$ Peter Thomas $^{2,4,5} \cdot$ Annegret Dahlmann-Noor $\mathbb{D}^{2,4,5}$
}

Received: 25 September 2018 / Accepted: 4 October 2018 / Published online: 23 October 2018

(c) The Royal College of Ophthalmologists 2018

Cycloplegic retinoscopy is the gold standard refraction approach in children. Various protocols are in use to achieve cycloplegia during outpatient appointments. Many use not only the muscarinergic antagonist, cyclopentolate (CP), which paralyses the ciliary muscle, but also the alphaadrenergic agonist, phenylephrine (PE), which as a mydriatic paralyses the iris constrictor, but has no effect on the ciliary muscle. Whilst mydriasis facilitates visualisation of the retinoscopy reflex, there is a risk of underestimating hypermetropia. Published evidence suggests that repeated

This work was presented at the annual meeting of the British Isles Paediatric Ophthalmology and Strabismus Association, Manchester, October 3-5, 2018.

\section{Annegret Dahlmann-Noor}

annegret.dahlmann-noor@nhs.net

Bedford Hospital Trust, Kempston Road, Bedford, UK

2 Moorfields at Bedford Hospital, Children's Eye Clinic, Enhanced Services Centre, Kimbolton Road, Bedford, UK

3 Cambridgeshire Community Services NHS Trust, Unit 3, Meadow Lane, St Ives PE27 4LG, UK

4 Paediatric Service, Moorfields Eye Hospital, 162 City Road, London EC1V 2PD, UK

5 NIHR Biomedical Research Centre at Moorfields Eye Hospital and UCL Institute of Ophthalmology, 162 City Road, London EC1V 2PD, UK instillation of $\mathrm{CP}$ only is effective even for brown and very dark irides [1-3].

To develop a Patient Group Directive (PGD) we carried out a two-cycle audit (CA18/PA/02). The first round evaluated our current protocol: blue iris, CP $1 \%$ once $(0.5 \%$ if age $<3$ months); brown iris, CP/PE $2.5 \%$ once; very dark iris, CP/PE twice, $10-15$ min apart; repeated if pupils still constrict on pentorch illumination. As standard, we set full dilation in $90 \%$ within $30 \mathrm{~min}$, i.e. the level expected for blue irides with a single $\mathrm{CP}$ drop [4].

In the second round, we only included children with brown or very dark iris, administering $\mathrm{CP}$ twice or three times, respectively, $10-15$ min apart.

Table 1 Ethnic background and iris colour of children assessed in this audit

\begin{tabular}{llllll}
\hline & \multicolumn{2}{l}{ Round 1 } & & \multicolumn{2}{l}{ Round 2 } \\
\cline { 6 - 6 } & $n$ & $\%$ & & $n$ & $\%$ \\
\hline Ethnicity & & & & \\
Afro-Caribbean & 5 & 9 & 10 & 20 \\
Asian & 7 & 13 & 19 & 37 \\
Caucasian & 44 & 79 & 20 & 39 \\
Chinese & & & 1 & 2 \\
Other & & & 1 & 2 \\
Iris colour & 32 & 57 & & \\
Blue & 11 & 20 & 14 & 27 \\
Brown & 13 & 23 & 37 & 73 \\
Very dark & & & & \\
\hline
\end{tabular}




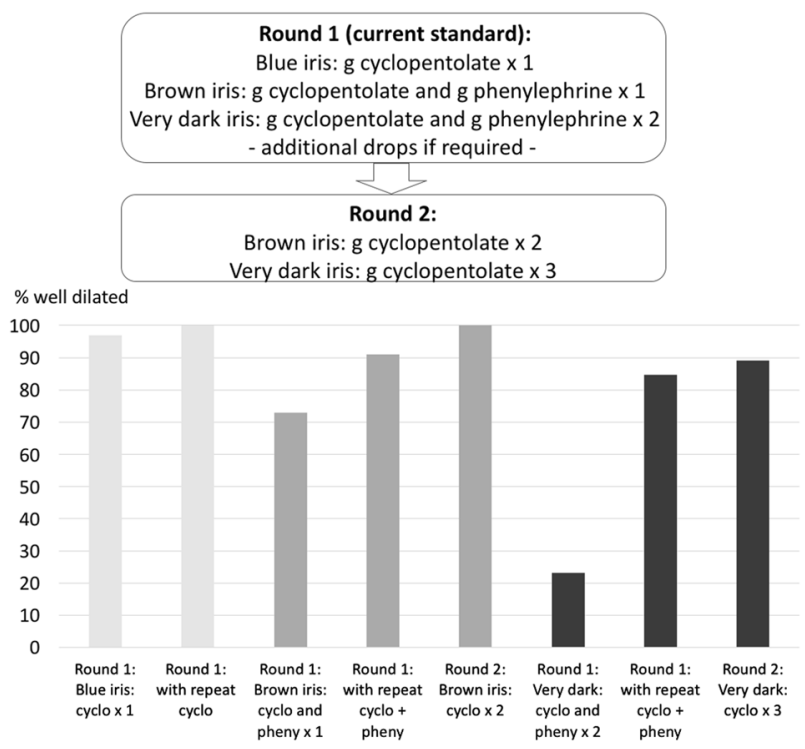

Fig. 1 Proportion of well-dilated pupils in rounds 1 and 2 of the audit. Eyes with blue irides are well dilated after one application of cyclopentolate $(\mathrm{CP})$. In brown irides, application of $\mathrm{CP}$ twice increases the proportion of well-dilated pupils, and in very dark irides, application of $\mathrm{CP}$ three times is effective. Phenylephrine does not appear to enhance this effect

We assessed pupil constriction to pentorch illumination, standard when deciding whether additional drops are required. We also noted time from instillation of the first drop to pentorch test.

We evaluated 107 consecutive children (Table 1). Round 1 found that pupils were dilated in $97 \%$ of children with blue, $73 \%$ with brown, and 23\% with very dark iris (Fig. 1), at a mean 24 min (SD 18) after first drop. Repeat instillation increased success to $100 \%, 91 \%$, and $85 \%$, respectively. In the second round, pupil dilation was achieved in all children with brown iris, and in $89 \%$ with very dark iris, at a mean 32 min (SD 13) after first instillation.

Our use of pupil constriction as outcome measure, which assesses mydriasis, not cycloplegia, is a limitation. However, as we found that better dilation is achieved without the use of PE, this audit has changed our practice and contributed positively to the development of the PGD.

Acknowledgements This work was supported by the National Institute for Health Research (NIHR) Moorfields Biomedical Research Centre. The views expressed are those of the authors and not necessarily those of the NHS, the NIHR or the Department of Health.

\section{Compliance with ethical standards}

Conflict of interest The authors declare that they have no conflict of interest.

\section{References}

1. Farhood Q. Cycloplegic refraction in children with cyclopentolate versus atropine. J Clin Exp Ophthalmol. 2012;3:239.

2. Ebri A, Kuper H, Wedner S. Cost-effectiveness of cycloplegic agents: results of a randomized controlled trial in nigerian children. Invest Ophthalmol Vis Sci. 2007;48:1025-31.

3. Celebi S, Aykan U. The comparison of cyclopentolate and atropine in patients with refractive accommodative esotropia by means of retinoscopy, autorefractometry and biometric lens thickness. Acta Ophthalmol Scand. 1999;77:426-9.

4. Zurevinsky J, Sawchuk K, Lim HJ, Lee CH, Rubab S. A clinical randomized trial comparing the cycloplegic effect of cyclopentolate drops applied to closed eyelids versus open eyelids. Am Orthopt J. 2016;66:114-21. 